\section{Knowledge and cultural beliefs of mothers regarding the risk factors of infant hearing loss and awareness of audiology services}

\section{Samantha M. Govender, Nasim Banu Khan}

Department of Audiology, University of KwaZulu-Natal, Durban, South Africa

\section{Abstract}

The aim of the paper is to describe the knowledge of mothers in Durban, South Africa, regarding risk factors of hearing loss in infants and their awareness of audiology services, and to describe their cultural beliefs about the risk factors for hearing loss in infants. A descriptive survey design with quantitative methods of analysis were used. Conveniently sampled mothers $(n=102)$ receiving postnatal care for their infants from eight provincial clinics within Durban consented to participate, yielding a response rate of $48 \%$. A questionnaire was used to collect the data and the Cronbach $\alpha$ was calculated yielding a score of 0.835 , indicating good internal consistency and reliability of the questionnaire. Sixty percent of the mothers were aware of risk factors, such as middle ear infections, ototoxic medication and consumption of alcohol during pregnancy. Seventy percent were unaware that NICU/mechanical ventilation for more than 5 days, prematurity, rubella and jaundice are considered risk factors for hearing loss, implying a need to create awareness amongst mothers regarding such risk factors. Sixty percent $(n=62)$ believed that bewitchment and ancestral curses can cause hearing loss. Cultural beliefs were associated with hearing loss, therefore, health professionals need to demonstrate cultural competence when providing audiology services, especially in a culturally and linguistically diverse countries such as South Africa. Although the mothers had an average knowledge about risk factors, two thirds did not know which professional to seek help from. There is a need to create awareness amongst mothers regarding the risk factors of infant hearing loss as well as audiology services in order to facilitate early detection and intervention. There is a need for health professionals to demonstrate cultural competence when working with their patients.

\section{Introduction}

The prevalence of permanent childhood hearing loss (PCHL) is 6 per 1000 live births in developing countries compared to 2 per 1000 live births in developed countries. ${ }^{1,2}$ In South Africa (SA), it is estimated that approximately 6116 babies are either born with or acquire hearing loss during the first weeks of life annually. A study conducted by Mahomed et al. ${ }^{3}$ investigated the prevalence of Sensory-Neural Hearing Loss (SNHL) in African children and found the prevalence to be $1.8 \%$ for those between the ages of 1-12 years. Undetected hearing loss has negative consequences for a child's speech, language, emotional and social development. ${ }^{4}$ Early Detection and Hearing Intervention (EDHI) services to manage hearing loss, including hearing aid fittings, aural rehabilitation and language stimulation amongst others, will provide the child with optimal opportunity to reach their full developmental potential. ${ }^{5,6}$

According to the Health Professionals Council of South Africa (HPCSA), ${ }^{5}$ all audiologic and medical evaluations to confirm the presence of hearing loss should be done by no later than four months of age, with the child enrolled to receive intervention services before eight months. However, findings from a study in the Western Cape Province indicated that the average age of diagnosis and intervention of children with PCHL was two years, with enrolment to intervention programs at 2.5 years. ${ }^{7}$ In Gauteng Province, it was found that the average age of diagnosis was 31 months, with hearing aid fittings at 39 months, and enrolment into early intervention services at 43 months. The findings of these studies indicate that detecting hearing loss in infants is generally delayed, thus influencing timeous interventions. As the early detection of hearing loss in South Africa remains primarily passive, parental concern about observed developmental delays is essential to identifying and managing hearing loss. ${ }^{8-10}$

The lack of mandatory neonatal and infant screening programs in South Africa provides impetus for parents to have sufficient knowledge about the risk factors of infant hearing loss. The decisions taken by mothers to routinely screen their children for hearing loss, or those taken after identifying a hearing loss, are important. Such decisions are based on their knowledge of and attitudes to the causes of infant hearing loss, as well as the available treatment options. ${ }^{11,12}$ However, due to limited access to information and health services in South Africa, mothers may not be aware of the
Correspondence: Samantha Marlanie Govender, Unit 11 Cannington square, 24 Ryde Avenue, Glenwood, Durban, South Africa.

Tel.: +27.073.499.0703

E-mail: samantha.govender@smu.ac.za

Key words: Competence; early hearing detection and intervention; newborn hearing screening.

Contributions: the authors contributed equally

Conflict of interest: the authors declare no potential conflict of interest.

Received for publication: 5 June 2016.

Revision received: 9 June 2017.

Accepted for publication: 10 June 2017.

This work is licensed under a Creative Commons Attribution NonCommercial 4.0 License (CC BY-NC 4.0).

CCopyright S.M. Govender and N.B. Khan, 2017 Licensee PAGEPress, Italy

Journal of Public Health in Africa 2017; 8:557 doi:10.4081/jphia.2017.557

risk factors associated with childhood hearing loss. Although EDHI programs have been initiated throughout the public sector health system?, implementation has been limited, mainly due to the government's priority to fight infectious diseases, with services being restricted by limited resources, socio-economic and health care barriers. ${ }^{7,13}$ In view of the various factors that can affect mothers taking advantage of EDHI programs, they should be made aware of the risk factors associated to infant hearing loss, ${ }^{14}$ as well as the audiology services available to manage it.

The Joint Committee on Infant Hearing (JCIH), a body of professional associations in pediatric healthcare in the United States of America, compiled a list of risk indicators of hearing loss from birth to 28 days and for targeted screening, which involves screening those individuals that present with high risk indicators for hearing loss. ${ }^{5,15-19}$ Other risk factors of hearing loss in infants are well established in the literature. ${ }^{5,15-19}$ These include, but are not limited to, craniofacial anomalies, syndromes, environmental risks, maternal infections, impact of HIV/AIDS, low birth weight, and an infant being in neonatal intensive care for more than five days. Treatments requiring assisted ventilation, exposure to ototoxic medications (gentamycin and tobramycin) or loop diuretics (furosemide/ Lasix) are additional risk factors. These contribute to $50 \%$ of cases of permanent childhood hearing loss, ${ }^{12}$ many of which 
may not be evident at birth and only manifest later. ${ }^{20}$ Additional risk factors relating to malaria and HIV infection and its treatment have been cited as context specific.

A study conducted in Pretoria by Swanepoel, Johl and Pienaar ${ }^{21}$ identified South African relevant risk factors to be admission to NICU for more than five days, asphyxia and low birth weight. Additional risk factors, as identified by Kanji and Khoza-Shangase, ${ }^{22}$ but excluded in the $\mathrm{JCIH}$ list, ${ }^{19}$ include prematurity and birth asphyxia. Furthermore, the prevalence of acquired disorders due to socio-economic conditions, such as otitis media, is a reality for many children in Africa. ${ }^{23}$ In a study conducted by Biagio Swanepoel, Laurent and Lundberg, ${ }^{24}$ regarding pediatric otitis media at a primary healthcare clinic, the prevalence of chronic suppurative otitis media (CSOM) was $6.6 \% .^{25}$ Given the impact that living conditions have on health, it is important for children to be screened for hearing loss in developing countries, where environmental health risks have an increased chance of causing hearing loss. In addition, screening and intervention services are not only constrained by limited human and financial resources, but are challenged by a lack of cultural awareness as well as culturally relevant and appropriate tools and resources. ${ }^{26}$ This highlights the need for professionals to engage with cultural beliefs about infant hearing loss so that contextually and culturally competent services can be provided.

Cultural competence is the awareness of the cultural factors that influences views and attitudes, and enables a health professional to work effectively in cross-cultural situations and address the clinical barriers to health care access, as experienced by those seeking it. ${ }^{27,28}$ Culturally appropriate health care is a term that indicates the need for health care professionals to deliver services that take the patients race, ethnic groups and cultural practices into account. ${ }^{29}$ Cultural beliefs can influence people's knowledge of health related conditions both in terms of their causative factors as well as their treatment and management plans. According to Kreuter and McClure, ${ }^{30}$ health programs can be customized and tailor made for cultural groups, provided that their cultural characteristics are understood. Culture influence people's attitudes to health conditions, the resulting decisions and treatment options, as well as their receptiveness to health care. ${ }^{28}$

ASHA describes the development of cultural competence as a dynamic and complex process, which requires a professional to understand their own culture in relation to other cultural belief systems. ${ }^{31}$ In doing that, professionals are able to expand their knowledge and thereby enhance their clinical skills. Health professionals therefore need to re-orientate themselves from a medical model of service delivery toward a biopsycho-social model, one which focuses more on community based services rather than institution based. This is in keeping with the primary health care framework, where promotion and prevention is favored over the curative approach to care. ${ }^{26}$ The primary health care (PHC) approach implies that intervention programs should be family and community centered, based on informed choices and recognition of and respect for cultural beliefs and traditions. ${ }^{5}$

Another factor that could influence mothers' knowledge of hearing loss relates to their access to audiology services. ${ }^{32}$ There is a shortage of audiologists as well as audiology related services in SA, especially within the public health sector, specifically in rural areas. ${ }^{33}$ This could limit access to information gained from such professionals regarding health conditions. According to a study by Swanepoel and Almec, ${ }^{12}$ access to audiology services was constrained due to lack of human resources. In the absence of the professionals, parents may thus not know whom to engage with regarding their concerns. Therefore, it is important to determine mothers' knowledge about the role of an audiologist and audiology service provision. In another published study it was studies revealed that there was overall poor knowledge regarding the profession of audiology. ${ }^{7}$ Knowledge regarding the role of an audiologist, the profession of audiology, as well as the access to audiological services is often limited, with more effort needing to be placed on promoting the profession. This is likely to improve public knowledge of hearing preservation, as well as its loss and management. Other possible reasons for mothers not accessing services, other than that of limited knowledge of risk factors, cultural beliefs and audiology service provision, are maternal levels of education, socio-economic levels, cultural and linguistic barriers, attitudes towards hearing screening and general lack of parental support for audiology services. ${ }^{34}$

\section{Materials and Methods}

\section{Aim}

The study aimed to establish mothers' understanding of the risk factors that contribute to infant hearing loss. The objectives of the study were to: i) determine participant's knowledge regarding risk factors and cultural beliefs that cause infant hearing loss; ii) determine participant's awareness of audiology services.

This was a descriptive survey design with quantitative methods of analysis. A self-administered questionnaire was used to obtain the data from mothers of infant children.

Participants who attended public sector antenatal clinics within the Durban central region for immunizations and other health related issues for their infants and young children were conveniently sampled, based on their availability and willingness to participate. A total of 250 mothers were approached to participate, of whom 102 took part in the study, yielding a response rate of $48 \%$. Participants were 18 years and older with a mean age of 24 , the youngest being 18 and the oldest 45

\section{Data collection and analysis}

Olusanya, Luxon and Wirz's ${ }^{11}$ questionnaire on risk factors of infant hearing loss was adapted for use in the present study. The questions relating to risk factors, awareness of audiology services and cultural beliefs were further informed by the following documents and articles: the $\mathrm{JCIH} ;{ }^{18}$ Northern and Downs; ${ }^{15}$ Nzama and Ntshalintshali, ${ }^{35}$ and Swanepoel and Almec. ${ }^{12}$ The self-administered questionnaire contained 15 questions on risk factors of hearing loss amongst infants, two questions on cultural beliefs, and four questions about knowledge and awareness of audiology services provided at public sector facilities. Each question had three possible responses, yes, no or uncertain. The questionnaires were available in English and isiZulu, with assistance being provided by participants when required.

Ethical clearance was obtained from the School of Health Science's Research Ethics Committee (SHSREC 024/12) at the University of KwaZulu-Natal, and gatekeeper permission was obtained from the Department of Health as well as from clinic managers. A pilot study was conducted on ten mothers to assess the reliability of the questionnaire. Adverts were placed in the waiting area to inform clinic patients of the study, and presentations were made to those waiting to be seen by clinic staff. Each interested participant was given an information sheet detailing the aims of the study and a consent form. Once the consent form was signed, participants completed their questionnaires in a cubicle outside the waiting area at the clinics. The data was first entered into an excel spreadsheet and descriptively analyzed using SPSS version 21 for frequency, percentage, mean and median scores. Upon completion of the questionnaire, informational pamphlet on risk factors for,, and signs and symptoms of 
hearing loss in children were provided to the mothers, as well as details of referral sources.

The Cronbach alpha score was also calculated to determine the internal consistency of the questionnaire and a score of 0.835 was obtained, which is above the normative value of 0.7 , indicating that the internal consistency of the questionnaire was significantly high. Data was analyzed using frequency counts.

\section{Results}

The results are presented with respect to the two objectives.

\section{Mother's knowledge regarding risk factors and cultural beliefs that cause infant hearing loss}

The results of the 15 risk factors and 2 cultural beliefs questions indicate that of the 102 participants, $53.9 \% \quad(n=55)$ were unaware that maternal rubella can cause hearing loss in infants (Table 1). With regard to low birth weight, prematurity and jaundice, nearly three quarters of the participants were unaware that these conditions could cause hearing loss in their infants. Eighty participants (78.4\%) were aware that ototoxic medication consumed by their child and middle ear infections (81.37\%) can result in hearing loss. With regard to cultural beliefs, angry ancestors were noted by $62(60.8 \%)$ and curses by $57(55.9 \%)$ of participants as causes of hearing loss on their infants.

\section{Mother's awareness of audiology services}

In terms of screening at birth, it was encouraging to note that the majority $(n=90$, $89.2 \%$ ) were aware that hearing could be screened at birth. However, only $45 \%$ $(n=46)$ were aware that an audiologist is the profession responsible for the screening, assessing, diagnosing and managing hearing loss in children and adults. Some participants thought that nurses and doctors were responsible for these services. More than $70 \%$ were unaware that an audiologist prescribes and fits hearing aids and can provide aural rehabilitation services.

\section{Discussion}

Early identification and intervention is best achieved when mothers' are aware of conditions that could occur either before, during or after birth that may place their infant at risk for developing hearing loss. Regarding the family related factors, more than half of the participants were unaware that maternal rubella can cause hearing loss. This was similar to a study by Swanepoel and Almec, ${ }^{12}$ where $43 \%$ of mothers identified maternal Rubella as a risk factor for hearing loss, but different to one by Olusanya, Luxon and Wirz in Nigeria, ${ }^{11}$ where $73 \%$ of mothers were aware of this risk factor. While maternal rubella is a rare occurrence globally, it still occurs in South Africa, and can cause systemic problems for the fetus, including hearing loss. ${ }^{37,38}$ Children with a suspected history or exposure to rubella need to have hearing assessments until at least 24 months of age. ${ }^{15}$ It is therefore important for mothers to know about this risk factor and how to important it is to have their child's hearing tested.

Regarding infant related factors, $50 \%$ of the mothers displayed poor knowledge regarding low birth weight as a risk factor for infant hearing loss. Low birth weight is specifically relevant in in low- and lower middle-income countries due to poverty related factors and excessive alcohol consumption. ${ }^{39}$ This is also a result of poor educational, unemployment and low income levels, as well as limited access to antenatal care in developing countries. ${ }^{40}$ Engdahl and Eskild ${ }^{41}$ in a Norwegian study found that birth weight of less than 1500 grams was associated with sensorineural hearing loss and that the risk of SensoryNeural Hearing Loss (SNHL) decreased with increasing birth weight. This demonstrates the need for audiological evaluations as soon as possible to identify problems. ${ }^{42}$ Greater awareness regarding how to prevent low birth weight and the associated increased risk of developing hearing loss needs to be created amongst mothers.

The findings relating to prematurity, and jaundice requiring exchange blood transfusion, are consistent with other South

Table 1. Mothers knowledge of risk factors and cultural beliefs that can affect childhood hearing loss.

\begin{tabular}{|c|c|c|c|}
\hline Risk factors & \multicolumn{3}{|c|}{ Responses $(\mathrm{N}=102)$} \\
\hline \multicolumn{4}{|c|}{ Knowledge of risk factors } \\
\hline $\begin{array}{l}\text { Family related factors } \\
\text { Hereditary conditions/family history of hearing loss } \\
\text { Maternal infections e.g. rubella } \\
\text { Consumption of alcohol during pregnancy } \\
\text { Rhesus incompatibility }\end{array}$ & $\begin{array}{l}55(53.92) \\
16(15.68) \\
63(61.76) \\
32(31.37)\end{array}$ & $\begin{array}{l}33(32.35) \\
55(53.92) \\
26(25.49) \\
24(23.52)\end{array}$ & $\begin{array}{l}14(13.72) \\
31(30.39) \\
13(12.74) \\
46(45.09)\end{array}$ \\
\hline $\begin{array}{l}\text { Intrinsic factors: specifically related to the child } \\
\text { Syndromes associated with hearing loss } \\
\text { Low birth weight } \\
\text { Prematurity } \\
\text { High fevers } \\
\text { Jaundice requiring exchange transfusion } \\
\text { Defects to the head, neck and face } \\
\text { Meningitis }\end{array}$ & $\begin{array}{c}31(30.39) \\
27(26.47) \\
34(33.33) \\
14(13.72) \\
16(15.68) \\
56(54.90) \\
(42.15)\end{array}$ & $\begin{array}{l}24(23.52) \\
49(48.03) \\
57(55.88) \\
62(60.78) \\
72(70.58) \\
23(22.54) \\
31(30.39)\end{array}$ & $\begin{array}{l}47(46.07) \\
26(25.49) \\
11(10.78) \\
26(25.49) \\
14(13.72) \\
23(22.54) \\
28(27.45)\end{array}$ \\
\hline $\begin{array}{l}\text { Extrinsic factors: external to the child } \\
\text { Mechanical ventilation for more than } 5 \text { days/neonatal intensive care of more than } 5 \\
\text { Ototoxic medication } \\
\text { Recurrent/unmanaged middle ear infections } \\
\text { Head traumas that requires hospitalization }\end{array}$ & $\begin{array}{l}18(17.64) \\
80(78.43) \\
83(81.37) \\
59(57.84)\end{array}$ & $\begin{array}{c}41(40.19) \\
10(9.80) \\
9(8.82) \\
22(21.56)\end{array}$ & $\begin{array}{c}43(42.15) \\
12(11.76) \\
10(9.80) \\
21(20.58)\end{array}$ \\
\hline \multicolumn{4}{|l|}{ Cultural beliefs } \\
\hline $\begin{array}{l}\text { Angry ancestors } \\
\text { Ancestral curses }\end{array}$ & $\begin{array}{l}62(60.8) \\
57(55.9)\end{array}$ & $\begin{array}{c}30(29.4) \\
32(31)\end{array}$ & $\begin{array}{l}10(0.98) \\
13(12.7)\end{array}$ \\
\hline
\end{tabular}


African studies. ${ }^{32,22}$ Swanepoel, Johl and Pienaar, ${ }^{21}$ who focused on the nature of hearing loss and associated risk profile, found that hyperbilirubinemia was one of the most prevalent risk factor. Other risk factors included birth weight less than 1500 grams, syndromes present, congenital infection, craniofacial defect and bacterial meningitis. Olusanya, Luxon and Wirz ${ }^{11}$ attribute the poor knowledge displayed by mothers to the deficiencies in health education during the antenatal period.

Regarding extrinsic factors, a study by Bielecki, Horbulewicz and Wolan ${ }^{42}$ found that mothers of infants who presented with PCHL after being on mechanical ventilation in excess of five days, displayed poor knowledge relating to the associated risk of hearing loss. It was reassuring to note that most of the mothers were aware of the problems associated with taking toxic meds and inner ear infections.

The findings of the study were that many mothers lacked knowledge regarding risk factors of infant hearing loss. There is a need for mothers to become more aware of such risks, particularly in developing countries, where a range of factors can present risks to the infant. Emerging risk factors from developing countries, such as Nigeria, include maternal hypertensive disorders in pregnancy; non-elective caesarean delivery; unskilled attendant at delivery and infant under-nutrition. ${ }^{43}$ However, the exact mechanism of how these factors compromise hearing status in early infancy is not yet fully understood and therefore requires further research. Relating to the burden of disease, particularly in the developing country context, is the increase in consumption of ototoxic medication by both mothers and infants. The large number of study participants who were aware that ototoxic drugs could affect hearing was slightly better than the findings in the Swanepoel and Almec ${ }^{12}$ study of 2008 , where $69 \%$ of mothers had knowledge of this, while this was $50 \%$ in the Olunsanya, Wirz and Luxon study in 2005. ${ }^{11}$ Participants displayed good knowledge of middle ear infections as a risk factor for hearing loss, this possibly being due to the visual nature and frequent occurrence of such a condition, including otitis media (OM) with effusion in developing countries, ${ }^{44}$ which compromises the quality of life. ${ }^{45}$ The reasons for the increased burden of otitis media in developing countries relates to inadequate awareness of this condition, ${ }^{45}$ limited specialist care, poorer health and socioeconomic conditions, as well as the rising prevalence of HIV/AIDS. ${ }^{44}$ A large body of evidence suggests that poorly or unmanaged $\mathrm{OM}$ can lead to permanent hearing loss, ${ }^{24,25,44,46}$ making it important for mothers to identify this problem as early as possible in order to receive appropriate and timeous management.

Regarding ancestral risk factors, more than half of the participants believed that angry ancestors and ancestral curses can cause hearing loss. A similar study conducted by Swanepoel and Almec, ${ }^{12}$ that investigated maternal knowledge and attitudes towards infant hearing loss amongst a South African sample revealed that $57 \%$ of mothers' held at least one cultural belief (blood impurities $44 \%$ and bewitchment $29 \%$ ) regarding a possible cause of hearing loss. A study conducted by de Andrade and Ross $^{47}$ with black SA traditional healers reported that cultural beliefs are associated with illness, the causes of hearing loss being due to bewitchment by ancestral spirits. This requires audiologists to demonstrate cultural competence and provide culturally sensitive services, in conjunction with traditional healers, should the context permit.

In South Africa, where peoples' beliefs are buried deep within their cultures, it is important that health care professionals take cognizance of these cultural beliefs so that it may be incorporated into information dissemination. ${ }^{12,50}$ Furthermore, the manner in which parents are informed about hearing screening, available management options as well as who gives them the information is important. ${ }^{51}$ Recommendations that are associated with social stigmatization, or that offend cultural norms, could result in loss to follow-up. ${ }^{51}$ As an example, there is considerable stigma attached to taking relatively healthy young children for hearing testing. ${ }^{52}$ However, parental education has been found to be effective in modifying health seeking behavior and reducing non-compliance in developing contexts, despite low education and literacy levels. ${ }^{53,54}$ In addition, a lack of cultural sensitivity among health care professionals when people do seek care can influence disease detection and management. While conditions causing hearing loss in children are well known by the medical community, there are many cultural beliefs surrounding its causes that should be integrated into managing both the child and their families at the various stages of their interaction with health care professionals.

The literature indicates that mothers demonstrate a willingness to have their babies screened for deafness throughout their childhood?. ${ }^{3}$ As almost $80 \%$ of the South African population access health services from the public sector, hearing screening programs initiated at this level can facilitate early detection and intervention. According to Swanepoel, ${ }^{3}$ audiological services in the country's public health care sector are well-positioned to provide the necessary follow-up services, such as diagnostic assessments, and prescribing and fitting amplification devices, provided that mothers' are aware of such services. This awareness can be created through advocating for the professions to be located in facilities throughout the country to ensure universal access. A study conducted by Naidoo $^{55}$ concluded that more marketing and advertising of the profession is required by audiologists in order to increase and improve knowledge and awareness of the profession, together with its role of identifying and managing hearing loss.

A recommendation emerging from this study is that infants born at public hospitals who are discharged on the same day can still be screened for a range of infant conditions? at the 6 week immunization visits. ${ }^{3}$ The use of the Road to Health card to monitor development of hearing milestones would prove beneficial in terms of early identification as well as rehabilitation of infants who are at risk of hearing loss. Culturally appropriate materials need to be developed in order to accommodate the diverse needs of the population and the cultural factors that can affect their uptake of medical services?. ${ }^{56}$ These tools should then follow a process of standardization, validation as well as systematic evaluation. Research should be directed toward developing culturally sensitive guidelines, protocols and tools to ensure that the health providers are suitably informed and prepared when engaging with people from the country's various cultures.

\section{Conclusions}

Mothers play a central role in the health and wellbeing of their children. In order for them to make the correct health decisions on behalf of their children, it is important that they are knowledgeable about the health disorders during their childhood years. Hearing loss is one such disorder that mothers need to be aware of, particularly as it is subtle in nature. ${ }^{5}$ Mothers need to be informed about the potential impact that the various risk factors could present to their infants' hearing. As found in other studies, cultural beliefs were associated with causes of hearing loss amongst infants. It is therefore essential that health care professionals practice within a culturally appropriate model of care, and develop cultural competency to enable them to better understand their patients in terms of what they believe are the causes of hearing related conditions and to deliver appropriate services. More 
research is required in this area to develop evidence based guidelines, which will lead to best practice in culturally diverse country like South Africa, but only if the services are offered throughout the country. An awareness of the profession of audiology and audiology service provision among mothers is also a critical factor in facilitating the early identification and intervention for children with hearing loss in South Africa.

\section{Clinical and research implications}

The findings of the study indicated a need to create awareness amongst mothers about the risk factors of infant hearing loss. This can be achieved by developing information brochures, holding workshops and providing information through other culturally appropriate avenues. The study also highlighted the need for audiologists to provide a more culturally competent service that accommodates the patients' beliefs. However, to improve the generalizability of the results, the study needs to be conducted on a wider geographical area. In the interim, a way forward would be for healthcare professionals to receive training in this area through continued professional development activities. The findings of the study further indicate the need for advocacy of the profession of audiology in order for communities to be aware of the professional services available. Research is needed.

\section{Limitations}

The small sample size was attributed to time constraints and the availability of mothers at the clinic who were willing to participate, and may have affected the results, given the wide age range. Information pertaining to the education level of mothers and the number of children they had was not obtained, which could have affected the responses and allowed for correlations of variables to be made. The study was only done in one urban region (Durban), therefore influencing the generalizability of the study finding to other regions in the province and the country.

\section{References}

1. Olusanya BO. Neonatal hearing screening and intervention in resource-limited settings: an overview. Arch Dis Child 2012;97:654-9.

2. World Health Organisation. New-born and infant hearing screening. Current issues and guiding principles for action. Outcome of a WHO informal consultation held at WHO headquarters, Geneva, Switzerland. Available from: http://www.whi.int/pbd/deaftiess/en/

3. Jacobson J, Jacobson C. Evaluation of hearing loss in infants and young children. Pediatr Ann 2004;33:811-21.

4. Storbeck C, Pitman P. Early intervention services in South Africa: moving beyond hearing screening. Int J Audiol 2008;47:36-43.

5. Health Professions Council of South Africa (HPCSA). Early Hearing Detection and Intervention Programs in South Africa: Position Statement. Available from: http://www.hpcsa. co.za/downloads/speech_education/earl y_hearing_detection_statement.pdf.

6. Cone-Wesson B, Wunderlich J. Auditory evoked potentials from the cortex: audiology applications. Curr Opin Otolaryngol Head Neck Surg 2003:11:372-7.

7. Swanepoel DW, Storbeck C, Friedland P. Early hearing detection and intervention in South Africa. Int J Pediatr Otorhinolaryngol 2003;73:783-6.

8. Soetjiningsih PA. Role of mother's perceptions on their child development on early detection of developmental deviation. Paediatrica Indonesiana 2001;41; 264-7.

9. Rajagopalan R, Selvarajan HG, Rajendran A, Ninan B. Grandmothers' perspective on hearing loss in children and newborn hearing screening. Indian J Otol 2014;20:20-3.

10. Olusanya BO, Neumann KJ, Saunders JE. The global burden of disabling hearing impairment: a call to action. Bull World Health Organ 2014;92:367-73.

11. Olusanya B, Luxon,L, Wirz SL. Screening for early childhood hearing loss in Nigeria. J Med Screen 2005;12: 115-8.

12. Swanepoel D, Almec N. Maternal views on infant hearing loss and early intervention in a South African community. Int J Audiol 2008;47:S44-8.

13. Friderichs N, Swanepoel DW, Hall JW. Efficacy of a community-based infant hearing screening program utilizing existing clinic personnel in Western Cape, South Africa. Int J Pediatr Otorhinolaryngol 2012;76:552-9.

14. Khoza-Shanagse K, Michal G. Early intervention in audiology: exploring the current status from a developing country context. Br J Med Med Res 2014;11:2238-49.

15. Northern JL, Downs MP, eds. Hearing in children. 5th ed. Philadelphia: Williams \& Wilkins; 2002.

16. Joint Committee on Infant Hearing. Year 1990. Position statement: Joint Committee on Infant Hearing. Available from: http://www.jcih.org/JCIH1990.
17. Joint Committee on Infant Hearing. Year 1994 Position statement: Joint Committee on Infant Hearing. Available from: www.jcih.org/JCIH1994.

18. Joint Committee on Infant Hearing. Year 2000 Position statement: Joint Committee on Infant Hearing. Available from: www.jcih.org/JCIH2000.

19. Joint Committee on Infant Hearing. Year 2007 Position statement: Joint Committee on Infant Hearing. Available from: www.jcih.org/JCIH2007.

20. Roeser JR, Valente M. Audiology: diagnosis. New York: Thieme Inc.; 2007.

21. Swanepoel DW, Johl L, Pienaar D. Childhood hearing loss and risk profile in a South African population. Int J Pediatr Otorhinolaryngol 2013;77.

22. Kanji A, Khoza-Shangase K. The occurrence of high-risk factors for hearing loss in very low birth weight neonates: a retrospective exploratory study of targeted hearing screening. South Afr J Commun Disord 2012;59:37.

23. Tiedt NJ, Butler, IRT, Hallbauer UM, et al. Paediatric chronic suppurative otitis media in the Free State province: clinical and audiological features. South Afr Med J 2013;103.

24. Biagio L, Swanepoel DW, Laurent C, Lundberg T. Paediatric otitis media at a primary healthcare clinic in South Africa. South Afr Med J 2014;104:7534.

25. World Health Organization. Deafness and hearing impairment: fact sheet 2013. Available from: http://www. who.int/mediacentre/factsheets/fs300/e $n$

26. Pascoe M, Norman V. Contextually relevant resources in speech-language therapy and audiology in South Africa are there any? South Afr J Commun Disord 2011;58:2-5.

27. Lupton D. Medicine as culture: illness, disease and the body. 3rd ed. London: Sage publiactions; 2012.

28. Kreuter MW, McClure SM. The role of culture in health communication. Ann Rev Public Health 2004;25:439-55.

29. The Lancet Commissions. Culture and health. 2014. Available from: http://www.thelancet.com/commissions/neglect-of-culture-in-health.

30. Centers for Disease Control and Prevention. Promoting cultural sensitivity: a practical guide for tuberculosis programs that provide services to Hmong persons from Laos. Atlanta: US Department of Health and Human Services; 2008.

31. American Speech-Language-Hearing Association (ASHA). Cultural compe- 
tence. Available from: http://www. asha.org/prpprinttemplate.aspx?folderi $\mathrm{d}=8589935230$

32. Kanji A, Kara R. Pediatrics physicians referral of children aged 0-3 years for audiological evaluation in the public health care sector. Audiol Res 2013;3:e7.

33. Theunissen M, Swanepoel DW. Early hearing detection and intervention services in the public sector in South Africa. Int J Audiol 2008;47:S23-9.

34. Van der Spuy T, Pottas L. Infant hearing loss in South Africa: age of intervention and parental needs for support. Int J Audiol 2008;47:1.

35. Nzama N, Ntshalintshali T. Nurses Knowledge about hearing impairments in children in Swaziland. 2003. [In Press]

36. World Medical Association. World Medical Association declaration of Helsinki ethical principles for medical research involving human subjects. JAMA 2013;310:2191-4.

37. Givens KT, Lee DA, Jones T, Ilstrup DM. Congenital rubella syndrome: ophthalmic manifestations and associated systemic disorders. Br J Ophthalmol 1993;77:358-63.

38. Niedzielska G, Kątska E, Szymula D. Hearing defects in children born of mothers suffering from rubella in the first trimester of pregnancy. Int J Pediatr Otorhinolaryngol 2000;54:1-5.

39. WHO 2010. World health statistics. 2010. Available from: http://www.who.int/whosis/whostat/EN _WHS10_Full.pdf

40. Parker JD, Schoendorf, KC, Kiely, JL. Associations between measures of socioeconomic status and low birth weight, small for gestational age, and premature delivery in the United States. Ann Epidemiol 1994;4:271-8.

41. Engdahl B, Eskild A. Birthweight and the risk of childhood sensorineural hearing loss. Paediatr Perinat Epidemiol 2007;21:495-500.

42. Bielecki I, Horbulewicz A, Wolan T. Risk factors associated with hearing loss in infants-an analysis of 5282 referred neonates. Int $\mathrm{J}$ Pediatr Otorhinolaryngol 2011;75:925-30.

43. Korres S, Balatsouras D, Nikolopoulos $\mathrm{T}$, et al. Making universal new-born hearing screening a success. Int $\mathrm{J}$ Pediatr Otorhinolaryngol 2006;70:2416.

44. Vohr BR, Widen JE, Cone-Wesson B, et al. Identification of neonatal hearing impairment: Characteristics of infants in the neonatal intensive care unit and well-baby nursery. Ear Hearing 2001;21:373-82.

45. Olusanya, B.O. Marking targeted screening for infant hearing loss an effective option in less developed countries. Int J Otorhinolaryngol 2011;75:316-21.

46. Olusanya BO, Newton VE. Global burden of childhood hearing impairment and disease control priorities for developing countries. Lancet 2007;369:13147.

47. Roy E, Hasan KZ, Richards JL, et al. Burden and outcome of acute otitis media in rural Bangladesh. South Afr J Child Health 2012;6:4.

48. Monasta L, Ronfani L, Marchetti F, et al. Burden of disease caused by otitis media: Systematic review and global estimates. PLoS One 2012; 7:e36226.

49. Andrade VD, Ross E. Beliefs and practices of Black South African traditional healers regarding hearing impairment. Int J Audiol 2005;44:489-99.

50. Anderson LM, Scrimshaw SC, Fullilove MT, et al. Culturally competent healthcare systems: a systematic review. Am J Prev Med 2003;24:68-79.

51. Moodley S, Storbeck C. The role of the neonatal nurse in early hearing detection and intervention in South Africa. Prof Nurs Today 2012;16.

52. McPherson B, Olusanya B. Screening for hearing loss in developing countries. In: McPherson B, Brouillette R, Eds. Audiology in developing countries. New York: Nova Science Publishers; 2008. pp. 75-105

53. Olusanya BO, Akinyemi, O. Community-based infant hearing screening in a developing country: parental uptake of follow-up services. BMC Public Health 2009;9:66.

54. Wolf KE. Cultural competence in audiology. The ASHA leader. 2004. Available from: http://www.asha.org/ Publications/leader/2004/040413/f0404 13b2.htm

55. Naidoo T. Audiological practice and service delivery in South Africa. Masters of audiology. PhD dissertation. University of Witwatersrand, Gauteng, 2006.

56. Carter J, Lees J, Murira G, et al. Issues in the development of cross-cultural assessments of speech and language for children. Int $\mathrm{J}$ Lang Commun Disord 2004;40:385-401. 